Pacific Journal of Mathematics

THE UPPER ENVELOPE OF INVARIANT FUNCTIONAL 


\section{THE UPPER ENVELOPE OF INVARIANT FUNCTIONALS MAJORIZED BY AN INVARIANT WEIGHT}

\section{A. VAN DAELE}

Let $A$ be a $C *$-algebra, $G$ a group of *automorphisms of $A$ and $\varphi$ a $G$-invariant weight. Assume that $\varphi$ takes finite values on a dense subset of $A^{+}$. It is shown that there is a largest element among the $G$-invariant weights $\psi_{0}$ majorized by $\varphi$ and weakly adherent to the set of $G$-invariant continuous positive linear functionals majorized by $\psi_{0}$. Moreover this weight majorizes every $G$-invariant continuous positive linear functional majorized by $\varphi$. If $A$ is a von Neumann algebra it is sufficient to assume that $\varphi$ takes finite values on a $\sigma$ weakly dense subset of $A^{+}$to get a similar result for normal functionals. Further characterisations of this weight are given in terms of the representation associated with $\varphi$. This relation is then used to prove that if $\varphi$ is lower semicontinuous, the existence of $G$-invariant continuous positive linear functionals majorized by $\varphi$ is equivalent to the existence of fixed points in the associated Hilbert space $\mathscr{H}$ and representation of $G$ in $\mathscr{H}$.

Finally two examples are discussed.

1. Introduction and notations. Recently a great deal of information has been obtained about states on a $C^{*}$-algebra $A$, invariant under a group $G$ of *-automorphisms. Unfortunately the set of invariant states on $A$ can be very small, in some cases it may be empty. So one may ask for the existence of $G$-invariant linear functionals on $A$ which are eventually unbounded. The concept of unbounded linear functionals has been introduced in [1] and [8]. In this paper we will be concerned with weights as defined in [1] which are invariant under a group of *automorphisms. The theory of $G$-invariant weights must make it possible to give an unified treatment of the theory of $G$-invariant states and the theory of traces. Indeed a state is a special case of a weight and a trace is a weight invariant under the group of inner automorphisms. In this paper we will show that the study of invariant weights can essentially be devided in two parts. The first of them being related to the theory of invariant states, the second being more similar to that of traces.

This fact will be discussed in $\S 2$ where we construct to some $G$-invariant weights $\rho$ an other $G$-invariant weight $\psi_{0}$ with the property that $\psi_{0}$ is the largest weight majorized by $\phi$ which is the upper envelope of $G$-invariant continuous positive linear functionals. 
In $\S 3$ we will construct a $G$-invariant projection map of the set $\mathscr{F}$ of continuous positive linear functionals majorized by $\varphi$ onto the set $\mathscr{F}_{0}$ of $G$-invariant elements in $\mathscr{F}$. This mapping will be used in $\S 4$ where we give more properties of the weight $\psi_{0}$ constructed in $\S 2$. Among others we will give a necessary and sufficient condition for the existence of $G$-invariant continuous positive linear functionals majorized by $\varphi$ if the latter is lower semi-continuous. Finally in the last section we discuss two examples.

We recall some notions and results as they can be found in [1]. A weight on a $C^{*}$-algebra is a function $\varphi$ defined on $A^{+}$with values in $[0, \infty]$ satisfying the following conditions:

(i ) $\varphi(x+y)=\varphi(x)+\varphi(y)$ for all $x, y \in A^{+}$

(ii) $\varphi(\lambda x)=\lambda \varphi(x) \quad$ for all real numbers $\lambda \geqslant 0$ and $x \in A^{+}$ (we agree that $0 \cdot \infty=0$ ).

The set of elements $x \in A$ such that $\varphi^{\prime}\left(x^{*} x\right)<\infty$ is a left ideal $\mathfrak{N}$ in $A$ and the set of elements $x \in A^{+}$with $\varphi(x)<\infty$ is the positive part $\mathfrak{M}^{+}$of the subalgebra $\mathfrak{M}$ defined as $\mathfrak{R}^{*} \mathfrak{R}$. The norm closures $\overline{\mathfrak{N}}$ and $\overline{\mathfrak{M}}$ of respectively $\mathfrak{N}$ and $\mathfrak{M}$ satisfy the relation

$$
\overline{\mathfrak{M}}=\overline{\mathfrak{R} * \mathfrak{R}}=\overline{\mathfrak{R}} \cap \overline{\mathfrak{R}}^{*} .
$$

The subalgebra $\mathfrak{M}$ is spanned by its positive part and the restriction of $\varphi$ to $\mathfrak{M}^{+}$can be extended to a linear form on $\mathfrak{M}$, still denoted by $\varphi$. With $\varphi$ is associated a Hilbert space $\mathscr{H}$, a representation $\pi$ of $A$ and a mapping $\Lambda$ of $\mathfrak{N}$ into $\mathscr{H}$ such that

(i) $\mathscr{A N}$ in dense in $\mathscr{H}$

(ii) $\varphi\left(x^{*} y\right)=(\Lambda y, \Lambda x)$ for all $x, y \in \mathfrak{N}$

(iii) $\pi(x) \Lambda y=\Lambda x y \quad$ for $x \in A$ and $y \in \Re$.

Throughout the paper we will be concerned with a fixed weight $\varphi$ so that it is unnecessary to write $\mathfrak{R}_{\varphi}, \mathfrak{M}_{\varphi}$ etc.; if we are given also another weight $\psi$ we will write $\mathfrak{R}_{\psi}, \mathfrak{M}_{\psi}, \cdots$ for the objects associated with $\psi$.

I would like to express my thanks to Prof. E. St $\phi$ rmer for his kind hospitality at the Mathematical Institute of the University of Oslo and for fruitful discussions. I am also indebted to Dr. N. H. Peterson for helpful comments and to Dr. F. Combes for discussions concerning the subject treated in this paper.

2. The upper envelope of invariant functionals majorized by an invariant weight. Let $A$ be a $C^{*}$-algebra and $G$ a group of *automorphisms of $A$. Fix a weight $\varphi$ on $A$ and assume that it 
is $G$-invariant i.e., $\varphi(g(x))=\varphi(x)$ for all $x \in A^{+}$and $g \in G$. As might be expected there is a unitary representation of $G$ in $\mathscr{H}$ that implements the automorphisms. The following lemma is more or less known (see [2] Lemma 4.7).

LEMma 2.1. Let $\phi$ be a G-invariant weight on $A$. Then $\mathfrak{M}$ and $\mathfrak{N}$ are $G$-invariant and there exists a unitary representation $\left\{U_{g}\right\}$ of $G$ in $\mathscr{H}$ such that
(i ) $U_{g} \Lambda x=\Lambda g(x)$
for $x \in \mathfrak{R}$
and $g \in G$
(ii) $U_{g} \pi(y) U_{g}^{-1}=\pi(g(y))$
for $y \in A$
and $g \in G$.

Proof. The invariance of $\mathfrak{R}$ and $\mathfrak{M}$ follows trivially from the invariance of $\varphi$. From $\|\Lambda g(x)\|^{2}=\varphi\left(g(x)^{*} g(x)\right)=\varphi\left(x^{*} x\right)=\|\Lambda x\|^{2}$ it follows that the mapping $\Lambda x \rightarrow \Lambda g(x)$ is well defined, continuous and can be extended to an isometry $U_{g}$ of $\mathscr{H}$. Clearly $U_{g-1} U_{g}=I$ so that $U_{g}$ is unitary. It follows from a trivial calculation that $\left\{U_{g}\right\}$ is a representation of $G$ and that the relations (i) and (ii) hold.

Definitions and notations 2.2. For any weight $\varphi$ on a $C^{*}$ algebra $A$ we will denote by $\mathscr{F}$ the family of continuous positive linear functionals majorized by $\varphi$, i.e., $f(z) \leqslant \varphi(z)$ for all $z \in A^{+}$and $f \in \mathscr{F}$. By $\mathscr{K}$ we denote the set of operators $S \in \pi(A)^{\prime}$ such that there is a positive real number $\lambda$ such that $\|S \Lambda x\| \leqslant \lambda\|x\|$ for all $x \in \Re$. If moreover $\varphi$ is $G$-invariant we denote by $\mathscr{F}_{0}$ and $\mathscr{K}_{0}$ respectively the $G$-invariant elements in $\mathscr{F}$ and $\mathscr{K}$. This makes sense if we let $G$ act on $\mathscr{F}$ and $\mathscr{C}$ in the natural way.

From the work of Combes [1] we may expect that $\mathscr{F}$ and $\mathscr{K}$, respectively $\mathscr{F}_{0}$ and $\mathscr{K}$., will be related to each other. We will clarify this relation without any restriction for the weight $\varphi$. Doing so we will be able to treat very general cases. The sets $\mathscr{F}, \mathscr{K}, \mathscr{F}_{0}$, and $\mathscr{K}_{0}$ and the relations we are going to prove in the next lemmas will be extensively used throughout the paper.

Lemma 2.3. $\mathscr{K}$ is a G-invariant left ideal in $\pi(A)^{\prime}$. For any $S \in \mathscr{K}$ there is a unique vector $\alpha$ in the closure of $\pi\left(\Re^{*}\right) \mathscr{H}$ such that $S \Lambda x=\pi(x) \alpha$ for all $x \in \mathfrak{N}$.

Proof. Take $S, S_{1}, S_{2} \in \mathscr{K}, T \in \pi(A)^{\prime}$ and $x \in \Re$. The relations

$$
\left\|\left(S_{1}+S_{2}\right) \Lambda x\right\| \leqslant\left\|S_{1} \Lambda x\right\|+\left\|S_{2} \Lambda x\right\|
$$

and

$$
\|T S \Lambda x\| \leqslant\|T\|\|S \Lambda x\|
$$

show that $\mathscr{K}$ is a left ideal. The relations $\left\|U_{g}^{-1} S U_{g} \Lambda x\right\|=\|S \Lambda g(x)\|$ 
and $\|g(x)\|=\|x\|$ for $g \in G$ show that $\mathscr{K}$ is invariant. Assume $\|S A x\| \leqq\|x\|$. Let $\left\{u_{k}\right\}$ be an approximate left identity in $\mathfrak{R}$ so that $\lim x u_{\lambda}=x$ for all $x \in \mathfrak{N}$ and therefore $\lim \pi(x) S \Lambda u_{\lambda}=\lim S \Lambda x u_{\lambda}=S \Lambda x$ because $S \in \mathscr{K}^{0}$. Let $p=\sum_{i=1}^{n} \pi\left(x_{i}\right)^{*} \gamma_{i}$ be an arbitrary element in $\pi\left(\mathfrak{R}^{*}\right) \mathscr{H}$. From the relation

$$
\sum_{i=1}^{n}\left(\gamma_{i}, S \Lambda x_{i}\right)=\lim \sum_{i=1}^{n}\left(\gamma_{i}, S \Lambda x_{i} u_{\lambda}\right)=\lim \left(p, S \Lambda u_{\lambda}\right)
$$

if follows that $\omega(p) \equiv \sum_{i=1}^{n}\left(\gamma_{i}, S \Lambda x_{i}\right)$ defines a linear functional $\omega$ on $\pi\left(\mathfrak{N}^{*}\right) \mathscr{H}$. Moreover

$$
|\omega(p)|=\lim \left|\left(p, S \Lambda u_{\lambda}\right)\right| \leqslant \lim \|p\|\left\|S \Lambda u_{\lambda}\right\| \leqslant\|p\|
$$

so that $\omega$ is continuous, can be extended to $\overline{\pi\left(\mathfrak{N}^{*}\right) \mathscr{H}}$ and that there exists a unique $\alpha \in \overline{\pi\left(\mathfrak{R}^{*}\right) \mathscr{H}}$ such that $\omega(p)=(p, \alpha)$. This means $(\gamma, S \Lambda x)=\left(\pi\left(x^{*}\right) \gamma, \alpha\right)$ for all $x \in \mathfrak{N}$ and $\gamma \in \mathscr{H}$ so that $S \Lambda x=$ $\pi(x) \alpha$ for all $x \in \mathfrak{N}$.

Lemma 2.4. $\mathscr{C}_{0}$ is a left ideal in the fixed point algebra of $\pi(A)^{\prime}$. For any $S \in \mathscr{K}_{0}$ there is a unique G-invariant vector $\alpha \in \overline{\pi\left(\mathfrak{N}^{*}\right) \mathscr{H}}$ such that $S \Lambda x=\pi(x) \alpha$ for all $x \in \Re$.

Proof. The first statement follows trivially from Lemma 2.3. By this lemma we have also the existence of $\alpha$ in $\overline{\pi\left(\mathfrak{R}^{*}\right) \mathscr{\mathscr { C }}}$ such that $S \Lambda x=\pi(x) \alpha$. As $S \in \mathscr{K}_{0}$ it is $G$-invariant and

$$
\begin{aligned}
S \Lambda x & =U_{g} S U_{g}^{-1} \Lambda x=U_{g} S \Lambda g^{-1}(x) \\
& =U_{g} \pi\left(g^{-1}(x)\right) \alpha=\pi(x) U_{g} \alpha
\end{aligned}
$$

by the use of Lemma 2.1. By invariance of $\mathfrak{R}$ we have that also $U_{g} \alpha \in \overline{\pi\left(\mathfrak{N}^{*}\right) \mathscr{H}}$ and by uniqueness that $U_{g} \alpha=\alpha$. This completes the proof.

REMARK 2.5. In the previous lemmas we gave a first characterisation of $\mathscr{K}$ and $\mathscr{K}_{0}$, in the next we will show the relation between $\mathscr{K}$ and $\mathscr{F}$, respectively $\mathscr{K}_{0}$ and $\mathscr{F}_{0}$. But first remark that $\mathscr{K}=\pi(A)^{\prime}$ implies that $I \in \mathscr{K}$ and so $\Lambda x=\pi(x) \alpha$ for some $\alpha \in \mathscr{H}$ so that

$$
\varphi\left(x^{*} x\right)=\left(\pi\left(x^{*} x\right) \alpha, \alpha\right) \quad \text { for all } x \in \mathfrak{R} \text {. }
$$

It follows that $\varphi$ coincides on $\mathfrak{M}^{+}$with a continuous positive linear functional. Conversely this property would imply that

$$
\varphi\left(x^{*} x\right)=\|\Lambda x\|^{2} \leqq \lambda\|x\|^{2}
$$

for some $\lambda>0$ so that $I \in \mathscr{K}$ and $\pi(A)^{\prime}=\mathscr{C}$. 
Lemma 2.6. For any $f \in \mathscr{F}$ there is a unique $S \in \mathscr{K}$ such that $0 \leqslant S \leqslant 1$ and $f\left(x^{*} x\right)=\|S \Lambda x\|^{2}$ for all $x \in \Re$. Conversely for any $S \in \mathscr{K}$ such that $\|S\| \leqslant 1$ there is a $f \in \mathscr{F}$ such that

$$
f\left(x^{*} x\right)=\|S \Lambda x\|^{2} \quad \text { for all } x \in \mathfrak{N} \text {. }
$$

Similarly for $\mathscr{F}_{0}$ and $\mathscr{K}_{0}$.

Proof. We prove the lemma for $\mathscr{F}_{0}$ and $\mathscr{K}_{0}$. Let $f \in \mathscr{F}_{0}$ then by ([1], Lemma 2.3) there is a $T_{f} \in \pi(A)^{\prime}$ such that $0 \leqslant T_{f} \leqslant 1$ and $f\left(x^{*} x\right)=\left(T_{f} \Lambda x, \Lambda x\right)$. Define $S=T_{f}^{1 / 2}$ then $0 \leqslant S \leqslant 1$ and $\|S \Lambda x\|^{2}=$ $f\left(x^{*} x\right) \leqslant\|f\|\|x\|^{2}$ so that $S \in \mathscr{K}$.

If $S^{\prime}$ is another element in $\pi(A)^{\prime}$ such that $0 \leqslant S^{\prime} \leqslant 1$ and $f\left(x^{*} x\right)=\left\|S^{\prime} \Lambda x\right\|^{2}$ then $\|S \Lambda x\|^{2}=\left\|S^{\prime} \Lambda x\right\|^{2}$ so that $S^{2}=S^{\prime 2}$ and by uniqueness of the square root that $S=S^{\prime}$. It then follows from the invariance of $f$ that $S$ is also $G$-invariant so that $S \in \mathscr{K}_{0}$. Conversely let $S \in \mathscr{K}_{0}$ such that $\|S\| \leqq 1$. By Lemma 2.4 there is a $G$-invariant $\alpha \in \mathscr{H}$ such that $S \Lambda x=\pi(x) \alpha$. A trivial calculation shows that $f$ defined by $f(z)=(\pi(z) \alpha, \alpha)$ for $z \in A$ is in $\mathscr{F}_{0}$ and satisfies the required relation.

We will proceed in the same way as in the proof of Proposition 13.11 of [10] in order to construct a largest $G$-invariant weight $\psi_{0}$ majorized by $\varphi$ and with the property that it is the upper envelope of $G$-invariant continuous positive linear functionals. Therefore we will need a property of $\mathscr{F}_{0}$ called " $\varepsilon$-filtrating" by Combes [1]. The following result can be found in ([4], Lemma 3.1) and is due to Dixmier. For sake of completeness we write down the short proof given there.

Lemma 2.7. Let $N$ be a left ideal in a von Neumann algebra $M$. For any two elements $S_{1}, S_{2}$ in the unit ball of $N$ and $\varepsilon>0$ there is a $S \in N$ such that

$$
(1-\varepsilon) S_{i}^{*} S_{i} \leqslant S^{*} S \leqslant 1 \quad \text { for } i=1,2 .
$$

Proof. Put

$$
\begin{aligned}
T_{i} & =(1-\varepsilon)\left(1-(1-\varepsilon) S_{i}^{*} S_{i}\right)^{-1} S_{i}^{*} S_{i} \quad \text { for } i=1,2 \\
T & =T_{1}+T_{2} \\
S & =(1+T)^{-1 / 2} T^{1 / 2}
\end{aligned}
$$

We will show that $S$ is the desired element. First a trivial calculation shows that $(1-\varepsilon) S_{i}^{*} S_{i}=1-\left(1+T_{i}\right)^{-1}$ and $S^{*} S=1-(1+T)^{-1}$ 
so that $(1-\varepsilon) S_{i}^{*} S_{i} \leqq S^{*} S \leqq 1$. Clearly $T_{i} \in N^{*} N$ since $N$ is a left ideal, so $T \in N^{*} N$ and by ([2], Lemma 4.11) $T^{1 / 2} \in N$ and therefore also $S \in N$.

We can now prove our first main result.

TheORem 2.8. Let $A$ be a $C^{*}$-algebra, $G$ a group of *automorphisms of $A$ and $\varphi$ a G-invariant weight on $A^{+}$such that $\mathfrak{M}$ is norm dense in $A$. There exists a largest G-invariant weight $\psi_{0}$ majorized by $\phi$ such that $\psi_{0}$ is the upper envelope of a family of G-invariant continuous positive linear functionals on $A$. Moreover $\psi_{0}$ majorizes every G-invariant continuous positive linear functional majorized by $\varphi$.

Proof. Define the function $\psi_{0}$ on $A^{+}$by

$$
\psi_{0}(x)=\sup \left\{f(x), f \in \mathscr{F}_{0}\right\} .
$$

It follows directly from the definition that $\psi_{0}(\lambda x)=\lambda \psi_{0}(x)$ for all real $\lambda>0$ and that $\psi_{0}\left(x_{1}+x_{2}\right) \leqslant \psi_{0}\left(x_{1}\right)+\psi_{0}\left(x_{2}\right)$ for all $x_{1}, x_{2} \in A^{+}$. We claim that also $\psi_{0}\left(x_{1}+x_{2}\right) \geqslant \psi_{0}\left(x_{1}\right)+\psi_{0}\left(x_{2}\right)$ so that $\psi_{0}$ is a weight on $A^{+}$.

Suppose first that $\psi_{0}\left(x_{1}\right)=\infty$, then for every integer $n$ there is a $f \in \mathscr{F}_{0}$ such that $f\left(x_{1}\right)>n$ and so $\psi_{0}\left(x_{1}+x_{2}\right) \geqslant f\left(x_{1}+x_{2}\right)>n$. We get $\psi_{0}\left(x_{1}+x_{2}\right)=\infty$. So we may suppose that $\psi_{0}\left(x_{1}\right)$ and $\psi_{0}\left(x_{2}\right)$ are finite. For any $\varepsilon>0$ we find $f_{1}, f_{2} \in \mathscr{F}_{0}$ such that $\psi_{0}\left(x_{i}\right)-\varepsilon<f_{i}\left(x_{i}\right)$ for $i=1,2$. By Lemma 2.6 there exist operators $S_{i}$ in $\mathscr{K}_{0}$ such that $0 \leqslant S_{i} \leqslant 1$ and $f_{i}\left(y^{*} y\right)=\left\|S_{i} A y\right\|$ for all $y \in \Re$. By Lemma $2.4 \mathscr{K}_{0}$ is a left ideal in the fixed point algebra of $\pi(A)^{\prime}$ so we can apply Lemma 2.7 to get an $S \in \mathscr{K}_{0}$ such that $(1-\varepsilon) S_{i}^{*} S_{i} \leqslant S^{*} S \leqslant 1$. Again by Lemma 2.6 we find $f \in \mathscr{F}_{0}$ such that $f\left(y^{*} y\right)=\|S \Lambda y\|^{2}$ for all $y \in \mathfrak{R}$. It follows that $(1-\varepsilon) f_{i}\left(y^{*} y\right) \leqslant f\left(y^{*} y\right)$ and by continuity of $f_{i}$ and $f$ and the density of $\mathfrak{M}$ that

$$
(1-\varepsilon)\left(\psi_{0}\left(x_{i}\right)-\varepsilon\right) \leqslant(1-\varepsilon) f_{i}\left(x_{i}\right) \leqslant f\left(x_{i}\right) .
$$

Summing up we get

$$
(1-\varepsilon)\left(\psi_{0}\left(x_{1}\right)+\psi_{0}\left(x_{2}\right)-2 \varepsilon\right) \leqslant f\left(x_{1}+x_{2}\right) \leqslant \psi_{0}\left(x_{1}+x_{2}\right)
$$

and this holds for all $\varepsilon>0$ so that $\psi_{0}\left(x_{1}\right)+\psi_{0}\left(x_{2}\right) \leqslant \psi_{0}\left(x_{1}+x_{2}\right)$ and that $\psi_{0}$ is a weight.

As $\mathscr{F}_{0}$ is $G$-invariant, so is $\psi_{0}$. If $f \in \mathscr{F}_{0}$ then $f \leqslant \varphi$ so that $\psi_{0} \leqslant \varphi$. From the definition we have also that $\psi_{0}$ is the upper envelope of $G$-invariant continuous positive linear functionals. Finally suppose that $\psi_{1}$ is another $G$-invariant weight majorized by $\varphi$ with this 
property. So for any $x \in A^{+}$such that $\psi_{1}(x)<\infty$ there is a $G$ invariant continuous positive linear functional $f \leqslant \psi_{1}$ such that

$$
\psi_{1}(x)-f(x)<1 .
$$

But $\psi_{1} \leqslant \varphi$ implies $f \in \mathscr{F}_{0}$ and $f(x) \leqslant \psi_{0}(x)$ so that $\psi_{1}(x)<\psi_{0}(x)+1$ for all $x \in \mathfrak{M}_{\psi_{1}}$ and therefore $\psi_{1}(x) \leqslant \psi_{0}(x)$. A similar argument holds for the case $\psi_{1}(x)=\infty$. So the proof is complete.

Corollary 2.9. For any weight $\varphi$ on a $C^{*}$-algebra $A$ such that $\mathfrak{M}$ is dense in $A$ there exists a largest weight $\psi$ majorized by $\varphi$ and lower semi-continuous on $A^{+}$. This weight majorizes every functional in $\mathscr{F}$.

The corollary follows by taking for $G$ the group consisting only of the identity automorphism. It is an extension of Propsition 1.10 of [1]. We next will show an analogous result for a $\sigma$-weakly lower semi-continuous weight on a von Neumann algebra. It is almost a consequence of Theorem 2.8 .

Theorem. 2.10. Let $A$ be a von Neumann algebra, $G$ a group of *-automorphisms of $A$ and $\varphi$ a G-invariant $\sigma$-weakly lower semicontinuous weight on $A^{+}$such that $\mathfrak{M}$ is $\sigma$-weakly dense in $A$. There exists a largest G-invariant weight $\psi_{0}$ majorized by $\phi$ such that $\psi_{0}$ is the upper envelope of normal G-invariant positive linear functionals on $A$.

Proof. Define the function $\psi_{0}$ on $A^{+}$by

$$
\psi_{0}(x)=\sup \left\{f(x), f \in \mathscr{F}_{0} \text { and } f \text { is normal }\right\} \text {. }
$$

To prove that $\psi_{0}$ is a weight we can use the same argument as in Theorem 2.8. By ([10], Prop. 13.10) $\pi$ is normal and all elements $f \in \mathscr{F}_{0}$ constructed in Lemma 2.6 are of the form $\omega_{\alpha} \circ \pi$ and hence are normal. Further in this case we must use the $\sigma$-weakly density of $\mathfrak{M}$ in $A$ and the normality of $f_{i}$ and $f$ to get

$$
(1-\varepsilon) f_{i}\left(x_{i}\right) \leqslant f\left(x_{i}\right) \text {. }
$$

Apart from these two remarks the proof carries over completely.

Remarks 2.11. Theorem 2.10 reduces partly to Proposition 13.11 of [10] if $G$ consists only of the identity automorphism. The proofs are then almost the same.

Let $\varphi$ and $\psi_{0}$ be as in Theorem 2.8. Define the function $\psi_{1}$ on $A^{+}$by $\psi_{1}(x)=\varphi(x)-\psi_{0}(x)$ if $x \in \mathbb{M}^{+}$and $\psi_{1}(x)=\infty$ for $x \notin \mathfrak{M}^{+}$. Clearly 
$\psi_{1}$ is again a $G$-invariant weight with $\mathfrak{M}_{\psi_{1}}=\mathfrak{M}$ and $\psi_{1} \leqslant \varphi$. If $f$ is any $G$-invariant continuous positive linear functional majorized by $\psi_{1}$ then $f \in \mathscr{F}_{0}$ and $f \leqslant \psi_{0}$. So for all $x \in \mathfrak{M}^{+}, f(x) \leqslant \psi_{0}(x)$ and $f(x) \leqslant \psi_{1}(x)$ so that $2 f(x) \leqslant \varphi(x)$ and $2 f \leqslant \varphi$. Similarly $n f \leqslant \varphi$ for all positive integers $n$ so that $f(x)=0$ for $x \in \mathfrak{M}^{+}$and by continuity that $f=0$.

We conclude that a $G$-invariant weight $\varphi$ such that $\overline{\mathfrak{M}}=A$ can be decomposed into two $G$-invariant weights $\psi_{0}$ and $\psi_{1}$ such that

(i ) $\varphi(x)=\psi_{0}(x)+\psi_{1}(x)$ for $x \in A^{+}$

(ii) $\psi_{0}$ is the upper envelope of $G$-invariant continuous positive linear functionals.

(iii) $\psi_{1}$ majorizes no $G$-invariant continuous positive linear functional.

This result enables us to devide the theory of $G$-invariant weights into two parts. In the first case we may assume that the weight majorizes no $G$-invariant continuous positive linear functional, in the second case we may assume that the weight is the upper envelope of such functionals. It is clear that the last case will be treatable by the use of known results for G-invariant states.

In the next section we will construct a unique normal $G$-invariant projection map $\phi$ of the ultra-weak closure $\overline{\mathscr{K}}$ of $\mathscr{K}$ onto the ultraweak closure $\overline{\mathscr{K}_{0}}$ of $\mathscr{K}_{0}$. We follow closely the arguments of ([6] Theorem 1). We will have that $\phi$ is also a projection map of $\mathscr{K}$ onto $\mathscr{K}_{0}$ and of $\mathscr{K}^{*} \mathscr{K}$ onto $\mathscr{K}_{0}^{*} \mathscr{K}_{0}$. Therefore it will be possible to define a unique $G$-invariant projection map $\phi^{\prime}$ of $\mathscr{F}$ onto $\mathscr{F}_{0}$ that is $w^{*}$-continuous on bounded sets. The map $\phi$ will be used to prove more results on $\psi_{0}$ in $\S 4$.

\section{A G-invariant projection map of $\mathscr{F}$ onto $\mathscr{F}_{0}$.}

Notations 3.1. Let $\varphi$ be a $G$-invariant weight on $A$. We will denote by $E_{0}$ the projection onto the fixed points in $\mathscr{H}$. So we have $U_{g} E_{0}=E_{0}$ for all $g \in G$ and therefore also $E_{0} U_{g}=E_{0}$. Moreover there exists a net of convex combinations, which we denote by

$$
\left\{\sum_{g \in G} \lambda^{i}(g) U_{g}\right\}_{i \in I},
$$

converging strongly to $E_{0}$, ([9] sect. 144), ( $\lambda^{i}$ are functions on $G$ with values in $[0,1]$ such that $\lambda^{i}(g)=0$ except on a finite set and $\left.\sum_{g \in G} \lambda^{i}(g)=1\right)$. By $E_{1}$ we will denote the projection $\left[\pi(A) E_{0} \mathscr{H}\right]$; clearly $E_{1} \in \pi(A)^{\prime}$ and $E_{1}$ is $G$-invariant because $\pi(A)$ and $E_{0} \mathscr{\mathscr { C }}$ are so. 
Proposition 3.2. Let $\varphi$ be a G-invariant weight on A. There exists a unique normal G-invariant positive projection map $\phi$ of $\overline{\mathscr{K}}$ onto $\overline{\mathscr{K}}_{0}$, the ultra-weak closures of $\mathscr{K}$ and $\mathscr{K}_{0}$. We have

$$
\phi(S) E_{0}=E_{0} S E_{0} \quad \text { for any } S \in \overline{\mathscr{K}} \text {. }
$$

In particular $\phi(\mathscr{K})=\mathscr{K}_{0}$ and $\phi\left(\mathscr{K}^{*} \mathscr{K}\right)=\mathscr{K}_{0}^{*} \mathscr{K}_{0}$.

Proof. We first define $\phi$ on $\mathscr{K}$, then we prove strong continuity of $\phi$ on bounded sets and extend it to $\overline{\mathscr{K}}$. Let $S \in \mathscr{K}$, by Lemma 2.3 there is a vector $\alpha$ such that $S \Lambda x=\pi(x) \alpha$ for all $x \in \Re$. Let $\left\{\sum_{G} \lambda^{i}(g) U_{g}\right\}_{i \in I}$ be a net of convex combinations converging strongly to $E_{0}$. For all $x \in \mathfrak{R}$ and all $i \in I$ we have

$$
\begin{aligned}
\left(\sum_{g \in G} \lambda^{i}(g) U_{g} S U_{g}^{-1}\right) \Lambda x & =\sum_{g \in G} \lambda^{i}(g) U_{g} S \Lambda g^{-1}(x) \\
& =\sum_{g \in G} \lambda^{i}(g) U_{g} \pi\left(g^{-1}(x)\right) \alpha \\
& =\sum_{g \in G} \lambda^{i}(g) \pi(x) U_{g} \alpha \\
& =\pi(x)\left(\sum_{g \in G} \lambda^{i}(g) U_{g}\right) \alpha .
\end{aligned}
$$

Because the net $\left\{\sum \lambda^{i}(g) U_{g} S U_{g}^{-1}\right\}_{i \in I}$ is bounded, it then converges strongly to an operator $\phi(S) \in \pi(A)^{\prime}$ such that $\phi(S) \Lambda x=\pi(x) E_{0} \alpha$. So $\|\phi(S) \Lambda x\| \leqq\|x\|\left\|E_{0} \alpha\right\|$ and $\phi(S) \in \mathscr{K}$. As $E_{0} \alpha$ is $G$-invariant it follows by a similar calculation that $\phi(S)$ is $G$-invariant. Clearly $\phi$ is linear and positive. If $S \in \mathscr{K}_{0}$ then $U_{g} S U_{g}^{-1}=S$ for all $g \in G$ so that $\phi(S)=S$ and $\phi$ is a positive projection map of $\mathscr{K}$ onto $\mathscr{K}_{0}$. Now let $g \in G$ then $S \Lambda x=\pi(x) \alpha$ implies $U_{g} S U_{g}^{-1} \Lambda x=\pi(x) U_{g} \alpha$ and

$$
\phi\left(U_{g} S U_{g}^{-1}\right) \Lambda x=\pi(x) E_{0} U_{g} \alpha=\pi(x) E_{0} \alpha
$$

so that $\phi\left(U_{g} S U_{g}^{-1}\right)=\phi(S)$ and $\phi$ is $G$-invariant. We also have that

$$
\begin{aligned}
\phi(S) E_{0} & =\operatorname{str.} \lim _{i \in I} \sum_{g \in G} \lambda^{i}(g) U_{g} S U_{g}^{-1} E_{0} \\
& =\operatorname{str}_{i \in I} \lim _{g \in G} \lambda^{i}(g) U_{g} S E_{0} \\
& =E_{0} S E_{0}
\end{aligned}
$$

Using this last relation we prove that $\phi$ is strongly continuous on bounded sets. Take $S_{0} \in \mathscr{K}_{0}$, by Lemma 2.4 there is a $G$-invariant vector $\alpha_{0} \in \mathscr{H}$ such that $S_{0} \Lambda x=\pi(x) \alpha_{0}$. Let $S_{0}=U\left|S_{0}\right|$ be the polar decomposition of $S_{0}$. Then $\left|S_{0}\right| \Lambda x=U^{*} S_{0} \Lambda x=\pi(x) U^{*} \alpha_{0}$ and as $S_{0}$ is $G$-invariant, we have also that $U^{*}$ and $U^{*} \alpha_{0}$ are $G$-invariant. It follows that $E_{1}\left|S_{0}\right|=\left|S_{0}\right|$ and so $S_{0} E_{1}=S_{0}$. Now let $\gamma \in \mathscr{H}$, then $\phi(S) \gamma=\phi(S) E_{1} \gamma$ and for any $\varepsilon>0$ there is a vector 


$$
\sum_{i=1}^{n} T_{i} \gamma_{i} \in \pi(A) E_{0} \mathscr{H}
$$

such that $\left\|E_{1} \gamma-\sum_{i=1}^{n} T_{i} \gamma_{i}\right\|<\varepsilon$ where $T_{i} \in \pi(A)$ and $\gamma_{i} \in E_{0} \mathscr{H}$. So

$$
\begin{aligned}
\|\phi(S) \gamma\| & =\left\|\phi(S) E_{1} \gamma\right\| \leqslant\left\|\phi(S)\left(E_{1} \gamma-\sum_{i=1}^{n} T_{i} \gamma_{i}\right)\right\|+\left\|\phi(S) \sum_{i=1}^{n} T_{i} \gamma_{i}\right\| \\
& \leqslant \varepsilon\|\phi(S)\|+\sum_{i=1}^{n}\left\|T_{i}\right\|\left\|\phi(S) \gamma_{i}\right\| \\
& \leqslant \varepsilon\|S\|+\sum_{i=1}^{n}\left\|T_{i}\right\|\left\|\phi(S) \gamma_{i}\right\|
\end{aligned}
$$

But $\phi(S) \gamma_{i}=\phi(S) E_{0} \gamma_{i}=E_{0} S E_{0} \gamma_{i}=E_{0} S \gamma_{i}$ and therefore

$$
\|\phi(S) \gamma\| \leqslant \varepsilon\|S\|+\sum_{i=1}^{n}\left\|T_{i}\right\|\left\|S \gamma_{i}\right\| \text {. }
$$

Therefore if $S \in \mathscr{\mathscr { C }}$ satisfies the conditions:

$$
\|S\| \leqslant 1, \quad\left\|S \gamma_{i}\right\| \leqslant \varepsilon n^{-1}\left\|T_{i}\right\|^{-1}
$$

then $\|\phi(S) \gamma\| \leqslant 2 \varepsilon$. Thus $\phi$ is strongly continuous on bounded parts. So by continuity we can extend $\phi$ to the ultra-weak closure $\overline{\mathscr{K}}$ of $\mathscr{K}$. The extension is still denoted by $\dot{\phi}$. Clearly $\phi$ will be a $G$ invariant projection map of $\overline{\mathscr{K}}$ onto $\overline{\mathscr{K}_{0}}$ and still satisfy $\phi(S) E_{0}=$ $E_{0} S E_{0}$. We show that the extension is also positive. Therefore let $S \in \overline{\mathscr{K}}$ and $0 \leqslant S \leqslant 1$; if $F$ is the largest projection in $\overline{\mathscr{K}}$ we have $S=S F=F S F$. So $S \in F \pi(A)^{\prime} F=\overline{\mathscr{K}} * \overline{\mathscr{K}} . \quad$ By ([3] Lemma 2.2) $\mathscr{K}^{*} \mathscr{K}$ is ultra-weakly dense in $\overline{\mathscr{K}^{*}} \overline{\mathscr{K}}$ and by the Kaplansky density theorem we have that the unit ball of the hermitian part of $\mathscr{K}^{*} \mathscr{K}$ is strongly dense in the unit ball of the hermitian part of $\overline{\mathscr{K}^{*}} \overline{\mathscr{K}}$. So $S$ is strongly adherent to

$$
\left\{T \xi T \in \mathscr{K}^{*} \mathscr{K}, T=T^{*},\|T\| \leqslant 1\right\} .
$$

We then have that $S^{2}$ is strongly adherent to

$$
\left\{T^{2}\left\{T \in \mathscr{\varkappa}^{*} \mathscr{\varkappa}, T=T^{*},\|T\| \leqslant 1\right\}\right.
$$

and by the work of Kaplansky [7] that $S=\left(S^{2}\right)^{1 / 2}$ is strongly adherent to $\left\{|T| \xi T \in \mathscr{C}^{*} \mathscr{K}, T=T^{*},\|T\| \leqslant 1\right\}$. As $\mathscr{K}$ is a left ideal $T \in \mathscr{K}^{*} \mathscr{K}^{2}$ implies $T \in \mathscr{K}$ and $|T| \in \mathscr{K}$ so that $S$ is strongly adherent to $\{T \xi T \in \mathscr{K}, 0 \leqslant T \leqslant 1\}$. We may conclude that $\phi$ is positive and so that $\phi$ is a normal $G$-invariant positive projection map of $\overline{\mathscr{K}}$ onto $\overline{\mathscr{K}_{0}}$. The normality follows from ([5], Appendix II).

If $\phi_{1}$ is another normal $G$-invariant projection map of $\overline{\mathscr{K}}$ onto $\overline{\mathscr{K}_{0}}$ then for any $S \in \mathscr{C}$ 


$$
\phi_{1}\left(\sum_{g \in G} \lambda^{i}(g) U_{g} S U_{g}^{-1}\right)=\dot{\phi}_{1}(S)
$$

and by continuity $\dot{\phi}_{1}(\phi(S))=\phi_{1}(S)=\phi(S)$. So $\phi$ and $\phi_{1}$ coincide on $\mathscr{K}$ and therefore also on $\overline{\mathscr{K}}$.

To complete the proof we must show that $\phi\left(\mathscr{K}^{*} \mathscr{K}^{\prime}\right)=\mathscr{K}_{0}^{*} \mathscr{K}_{0}$. Take $S \in \mathscr{C}$ such that $\|S \Lambda x\| \leqslant\|x\|$ then

$$
\begin{aligned}
\left(\sum_{g \in G} \lambda^{i}(g) U_{g} S^{*} S U_{g}^{-1} \Lambda x, \Lambda x\right) & =\sum_{g \in G} \lambda^{i}(g)\left\|S U_{g}^{-1} \Lambda x\right\|^{2} \\
& =\sum_{g \in G} \lambda^{i}(g)\left\|S \Lambda g^{-1}(x)\right\|^{2} \\
& \leqslant \sum_{g \in G} \lambda^{i}(g)\|x\|^{2}=\|x\|^{2} .
\end{aligned}
$$

So that $\left(\phi\left(S^{*} S\right) \Lambda x, \Lambda x\right) \leqslant\|x\|^{2}$ and $\phi\left(S^{*} S\right)^{1 / 2} \in \mathscr{K}_{0}$ and $\phi\left(S^{*} S\right) \in \mathscr{K}_{0}^{*} \mathscr{K}_{0}$. As $\mathscr{K}^{*} \mathscr{K}$ is spanned by its positive elements and those elements are of the form $S^{*} S$ with $S \in \mathscr{K}$ ([2] Lemma 4.11) we get

$$
\phi\left(\mathscr{L}^{*} \mathscr{K}_{L}\right) \subseteq \mathscr{K}_{0} * \mathscr{K}_{0} .
$$

This completes the proof.

COROLLARY 3.3. Let $F$ and $F_{0}$ be the largest projections in respectively $\overline{\mathscr{K}}$ and $\overline{\mathscr{K}_{0}}$, the ultra-weak closures of $\mathscr{K}_{\mathrm{r}}$ and $\mathscr{K}_{0}$, then $\phi(F)=F_{0}$.

Proof. As $F_{0} \in \overline{\mathscr{K}}$ we have $F_{0} \leqslant F$ and $F_{0}=\phi\left(F_{0}\right) \leqslant \phi(F)$. As $\phi(F) \in \overline{\mathscr{K}_{0}}$ and $0 \leqslant \phi(F) \leqslant 1$ we have $\phi(F) \leqslant F_{0}$ so that $\phi(F)=F_{0}$.

Proposition 3.4. Let $\varphi$ be a G-invariant weight on $A$. Then there exists a G-invariant projection map $\phi^{\prime}$ of $\mathscr{F}$ into $\mathscr{F}_{0}^{-}$satisfying $\phi^{\prime}\left(f_{1}+f_{2}\right)=\phi^{\prime}\left(f_{1}\right)+\phi^{\prime}\left(f_{2}\right)$ and $\phi^{\prime}(\lambda f)=\lambda \phi^{\prime}(f)$ for all $f, f_{1}, f_{2} \in \mathscr{F}$ and positive real numbers $\lambda$. If moreover $\mathfrak{M}$ is norm dense in $A$, then $\phi^{\prime}$ is onto $\mathscr{F}_{0}, w^{*}$-continuous on bounded sets and unique.

Proof. First we define $\dot{\phi}^{\prime}(f)$ for $f \in \mathscr{F}$. Given $f \in \mathscr{F}$ there is a unique $S \in \mathscr{K}$ such that $0 \leqslant S \leqslant 1$ and $f\left(x^{*} x\right)=\left(S^{*} S \Lambda x, \Lambda x\right)$ by Lemma 2.6. Then $\phi\left(S^{*} S\right) \in \mathscr{K}_{0}^{*} \mathscr{K}_{0}$ by Proposition 3.2 and

$$
\dot{\phi}\left(S^{*} S\right)^{1 / 2} \in \mathscr{K}_{0} \quad \text { by ([2] Lemma 4.11). }
$$

By Lemma 2.4 there is a unique invariant vector $\alpha \in \overline{\pi\left(\mathfrak{N}^{*}\right) \mathscr{H}}$ such that $\dot{\phi}\left(S^{*} S\right)^{1 / 2} \Lambda x=\pi(x) \alpha$ for all $x \in \mathfrak{N}$. Define $\phi^{\prime}(f)$ by

$$
\phi^{\prime}(f)(z)=\left(\pi(z) \alpha, \alpha^{\prime}\right)
$$

Clearly $\phi^{\prime}$ maps $\mathscr{F}$ into $\mathscr{F}_{0}$. 
Let $f_{1}, f_{2} \in \mathscr{F}$ such that $f_{1}=\lambda f_{2}$ for a real number $\lambda \geqslant 0$. It is clear that the corresponding $S_{1}, S_{2} \in \mathscr{K}$ satisfy $S_{1}=\lambda^{1 / 2} S_{2}$ and that $\alpha_{1}=\lambda^{1 / 2} \alpha_{2}$ for the corresponding vectors. Then $\phi^{\prime}\left(f_{1}\right)=\lambda \phi^{\prime}\left(f_{2}\right)$. Further suppose $f_{1}, f_{2}, f \in \mathscr{F}$ such that $f=f_{1}+f_{2}$. We then have

$$
S^{*} S=S_{1}^{*} S_{1}+S_{2}^{*} S_{2}
$$

for the corresponding elements in $\mathscr{K}$. By linearity of $\phi$ we get

$$
\phi\left(S^{*} S\right)=\phi\left(S_{1}^{*} S_{1}\right)+\phi\left(S_{2}{ }^{*} S_{2}\right) .
$$

As in the proof of ([5] Th. 1 p. 85) we can find operators

$$
U_{1}, U_{2} \in \pi(A)^{\prime}
$$

such that

$$
\phi\left(S_{i}^{*} S_{i}\right)^{1 / 2}=U_{i} \phi\left(S^{*} S\right)^{1 / 2} \quad \text { for } i=1,2
$$

and

$$
\left(U_{1}^{*} U_{1}+U_{2}^{*} U_{2}\right) \dot{\phi}\left(S^{*} S\right)^{1 / 2}=\dot{\phi}\left(S^{*} S\right)^{1 / 2} .
$$

Let $\alpha_{1}, \alpha_{2}, \alpha$ be the vectors in $\overline{\pi\left(\mathfrak{R}^{*}\right) \mathscr{H}}$ corresponding to $\phi\left(S_{1}{ }^{*} S_{1}\right)^{1 / 2}$, $\phi\left(S_{2}{ }^{*} S_{2}\right)^{1 / 2}$ and $\phi\left(S^{*} S\right)^{1 / 2}$. Then

$$
\begin{aligned}
\pi(x) \alpha_{i} & =\phi\left(S_{i}^{*} S_{i}\right)^{1 / 2} \Lambda x=U_{i} \phi\left(S^{*} S\right)^{1 / 2} \Lambda x \\
& =\pi(x) U_{i} \alpha .
\end{aligned}
$$

So that $\alpha_{i}=U_{i} \alpha$ by unicity and invariance of $\overline{\pi\left(\Re^{*}\right) \mathscr{H}}$. A similar argument shows that $\alpha=\left(U_{1}^{*} U_{1}+U_{2}^{*} U_{2}\right) \alpha$. We apply all this to find the following

$$
\begin{aligned}
{\left[\dot{\phi}^{\prime}\left(f_{1}\right)+\dot{\phi}^{\prime}\left(f_{2}\right)\right](z) } & =\left(\pi(z) \alpha_{1}, \alpha_{1}\right)+\left(\pi(z) \alpha_{2}, \alpha_{2}\right) \\
& =\left(\pi(z) \alpha, U_{1}^{*} U_{1} \alpha\right)+\left(\pi(z) \alpha, U_{2}^{*} U_{2} \alpha\right) \\
& =(\pi(z) \alpha, \alpha)=\phi^{\prime}(f)(z)
\end{aligned}
$$

for all $z \in A$, showing $\phi^{\prime}\left(f_{1}+f_{2}\right)=\phi^{\prime}\left(f_{1}\right)+\phi^{\prime}\left(f_{2}\right)$.

We next show that it is a projection map. Let $f$ be in the image of $\phi^{\prime}$. Then there exists a $S \in \mathscr{K}_{0}$ and a $G$-invariant vector $\alpha \in \overline{\pi\left(\mathfrak{R}^{*}\right) \mathscr{H}}$ such that $f\left(x^{*} x\right)=\left(S^{*} S \Lambda x, \Lambda x\right), S \Lambda x=\pi(x) \alpha$ for $x \in \mathfrak{R}$ and $f(z)=(\pi(z) \alpha, \alpha)$ for $z \in A$. Then $\phi^{\prime}\left(S^{*} S\right)=S^{*} S$ and $\phi^{\prime}(f)(z)=$ $(\pi(z) \alpha, \alpha)$ for $z \in A$. The $G$-invariance of $\phi^{\prime}$ follows straight forward from the $G$-invariance of $\phi$. So we proved the first part.

Assume now that $\mathfrak{M}$ is norm dense in $A$. If $f \in \mathscr{F}_{0}$ there exists a $S \in \mathscr{K}_{0}$ and an invariant vector $\alpha \in \overline{\pi\left(\mathfrak{N}^{*}\right) \mathscr{\mathscr { C }}}$ such that $f\left(x^{*} x\right)=$ $\left(S^{*} S \Lambda x, \Lambda x\right)$ and $S \Lambda x=\pi(x) \alpha$ for $x \in \mathfrak{R}$. Then $\phi\left(S^{*} S\right)=S^{*} S$ and 
$\phi^{\prime}(f)(z)=(\pi(z) \alpha, \alpha)$ for $z \in A$. But also $f\left(x^{*} x\right)=\left(\pi\left(x^{*} x\right) \alpha, \alpha\right)$ for $x \in \mathfrak{R}$ and it follows by the density of $\mathfrak{M}$ in $A$ that

$$
f(z)=(\pi(z) \alpha, \alpha)=\phi^{\prime}(f)(z)
$$

for all $z \in A$. So $\phi^{\prime}$ is a projection map onto $\mathscr{F}_{0}$. To complete the proof it remains to show that $\phi^{\prime}$ is $\omega^{*}$-continuous on bounded sets for then a similar argument as in Proposition 3.2 provides the uniqueness.

Let $f_{i}$ be a sequence in $\mathscr{F}$ converging to $f$ in the $w^{*}$-topology and such that $\left\|f_{i}\right\| \leqslant 1$. We must show that $\phi^{\prime}\left(f_{i}\right)(z)$ converges to $\phi^{\prime}(f)(z)$ for and $z \in A$. Denote by $S_{i}, S$ the corresponding elements in $\mathscr{K}$ and by $\alpha_{i}, \alpha$ the vectors in $\mathscr{K}$ corresponding to $\phi\left(S_{i}{ }^{*} S_{i}\right)^{1 / 2}$ and $\phi\left(S^{*} S\right)^{1 / 2}$. Take first $z=x^{*} x$ with $x \in \mathfrak{N}$. From the relations

$$
\begin{aligned}
\phi^{\prime}\left(f_{i}\right)\left(x^{*} x\right) & =\left(\phi\left(S_{i}{ }^{*} S_{i}\right) \Lambda x, \Lambda x\right) \\
f_{i}\left(x^{*} x\right) & =\left(S_{i}{ }^{*} S_{i} \Lambda x, \Lambda x\right),
\end{aligned}
$$

the corresponding once for $f$ and the normality of $\dot{\phi}$ we get that $\lim \phi^{\prime}\left(f_{i}\right)\left(x^{*} x\right)=\phi^{*}(f)\left(x^{*} x\right)$. As $\mathfrak{M}$ is linearly spanned by elements $x^{*} x$ with $x \in \mathfrak{N}$ we have that $\lim \phi^{\prime}\left(f_{i}\right)(z)=\phi^{\prime}(f)(z)$ for all $z \in \mathfrak{M}$. Then by the fact that $\left\|\phi^{\prime}\left(f_{i}\right)\right\| \leqslant\left\|f_{i}\right\| \leqslant 1$ and the density of $\mathfrak{M}$ this relation holds for all $z \in A$.

So the proof is complete.

4. More properties of the upper envelope of $\mathscr{F}_{0}$. In this section we will get some more information about the $G$-invariant weight $\psi_{0}$, constructed in Theorem 2.8 and from now on called the upper envelope of $\mathscr{F}_{0}$. We will also relate the existence of fixed points in $\mathscr{H}$ to the existence of nontrivial elements in $\mathscr{F}_{0}$. Finally we will consider the set of weights majorized by $\psi_{0}$. In this section again $F$ and $F_{0}$ will stand for the largest projections in the ultraweak closures $\overline{\mathscr{K}}$ and $\overline{\mathscr{K}_{0}}$ of resp. $\mathscr{K}$ and $\mathscr{K}_{0}$.

THEOREM 4.1. Let $\varphi$ be a G-invariant weight on $A$ such that $\mathfrak{M}$ is norm dense in $A$. Let $F_{0}$ be the largest projection in the ultraweak closure $\overline{\mathscr{K}_{0}}$ of $\mathscr{K}_{0}$ and $\psi_{0}$ the upper envelope of $\mathscr{F}_{0}$. Then $\psi_{0}\left(x^{*} x\right)=\left(F_{0} \Lambda x, \Lambda x\right)$ for all $x \in \Re$ 。

Proof. By Lemma 2.4, $\mathscr{K}_{0}$ is a left ideal in the fixed point algebra $\mathfrak{B}$ of $\pi(A)^{\prime}$. Then by ([2] Lemma 4.11) $\mathscr{K}_{0}^{*} \mathscr{K}_{0}$ is a facial subalgebra of $\mathfrak{B}$ and ultra-weak dense in $\overline{\mathscr{K}_{0}} * \overline{\mathscr{K}_{0}}=F_{0} \mathfrak{B} F_{0}$ by ([3] Lemma 2.2). So by ([3] Lemma 2.3) there is an increasing net $\left\{T_{i}\right\}_{i \in I}$ of positive elements in $\mathscr{K}_{0}^{*} \mathscr{K}_{0}$ tending ultra-weakly to $F_{0}$. Again by 
([2] Lemma 4.11) there exists a $S_{i} \in \mathscr{K}_{0}$ such that $T_{i}=S_{i}{ }^{*} S_{i}$ and by Lemma 2.6 there exists a $f_{i} \in \mathscr{F}_{0}$ such that $f_{i}\left(x^{*} x\right)=\left\|S_{i} \Lambda x\right\|$ for all $x \in \Re$. So $\left(F_{0} \Lambda x, \Lambda x\right)=\sup _{i \in I}\left(S_{i}{ }^{*} S_{i} \Lambda x, \Lambda x\right)=\sup _{i \in I} f_{i}\left(x^{*} x\right)$. But $f_{i} \in \mathscr{F}_{0}$ so by definition $f_{i} \leqslant \psi_{0}$ and $\left(F_{0} \Lambda x, \Lambda x\right) \leqslant \psi_{0}\left(x^{*} x\right)$. On the other hand if $f \in \mathscr{F}_{0}$ there exists a $S \in \mathscr{C}_{0}$ such that $0 \leqslant S \leqslant 1$ and $f\left(x^{*} x\right)=\|S \Lambda x\|^{2}$ by Lemma 2.6. This implies that $S * S \leqslant F_{0}$ and so that $f\left(x^{*} x\right) \leqslant\left(F_{0} \Lambda x, \Lambda x\right)$. It follows that also

$$
\psi_{0}\left(x^{*} x\right)=\sup _{f \in \mathscr{F}_{0}} f\left(x^{*} x\right) \leqslant\left(F_{0} \Lambda x, \Lambda x\right) .
$$

This completes the proof.

Corollary 4.2. Any weight $\varphi$ on $A$, such that $\overline{\mathfrak{M}}=A$, is lower semi-continuous on $\mathfrak{M}^{+}$if and only if $\mathscr{C}$ is ultra-weakly dense in $\pi(A)^{\prime}$.

Proof. Let $G$ be the group consisting only of the identity automorphism, then $\mathscr{F}_{0}=\mathscr{F}, \mathscr{K}_{0}=\mathscr{K}$ and $F=F_{0}$. If $\overline{\mathscr{K}}=\pi(A)^{\prime}$ then $F=I$ and $\psi_{0}\left(x^{*} x\right)=(\Lambda x, \Lambda x)=\varphi\left(x^{*} x\right)$. It follows that $\varphi$ is lower semi-continuous on $\mathfrak{M}^{+}$. If on the other hand $\varphi$ is lower semicontinuous on $\mathfrak{M}^{+}$, then by ([1], Prop. 1.7) $\varphi \mid \mathfrak{M}^{+}$is weakly adherent to $\mathscr{F}$ so that

$$
(\Lambda x, \Lambda x)=\varphi\left(x^{*} x\right)=\psi_{0}\left(x^{*} x\right)=\left(F_{0} \Lambda x, \Lambda x\right) .
$$

So that $F_{0}=F=I$ and $\overline{\mathscr{K}}=\pi(A)^{\prime}$.

COROLlary 4.3. Let $\Phi$ be a G-invariant weight on A, assume $\mathfrak{M}$ norm dense in $A$ and $\varphi$ lower semi-continuous on $\mathfrak{M}^{+}$. Then $F_{0}=\left[\pi(A) E_{0} \mathscr{H}\right]$. Moreover $\varnothing$ majorizes no nonzero G-invariant continuous positive linear functional iff $\mathscr{H}$ has no fixed points.

Proof. From the proof of Proposition 3.2 we know that for any $S \in \mathscr{K}_{0}$ we have $S_{0} E_{1}=S_{0}$ where $E_{1}=\left[\pi(A) E_{0} \mathscr{H}\right]$. By continuity we get $F_{0} \leqslant E_{1}$. From Corollary 4.2. we have $F=I$. So again from Proposition 3.2 and Corollary 3.3 we have

$$
F_{0} E_{0}=\phi(I) E_{0}=E_{0} I E_{0}=E_{0} .
$$

As $F_{0} \in \pi(A)^{\prime}$ we get $F_{0} T E_{0}=T E_{0}$ for all $T \in \pi(A)$ so that also $F_{0} \geqslant E_{1}$. The last statement then follows from the relations $\psi_{0}\left(x^{*} x\right)=\left(F_{0} \Lambda x, \Lambda x\right)$ and $F_{0}=\left[\pi(A) E_{0} \mathscr{H}\right]$.

Remark that the existence of nonzero elements in $\mathscr{F}_{0}$ implies trivially the existence of fixed points. The converse however is not 
so clear.

Corollary 4.4. Let $\varphi$ be a G-invariant weight on $A$ such that $\overline{\mathfrak{M}}=A$. Then there is an increasing net $\left\{f_{i}\right\}_{i \in I}$ in $\mathscr{F}_{0}$ such that $\psi_{0}(x)=\sup f_{i}(z)$ for all $z \in A^{+}$such that $\psi_{0}(z)<\infty$.

Proof. From the proof of the theorem there is an increasing net $\left\{f_{i}\right\}_{i \in I}$ in $\mathscr{F}_{0}$ such that

$$
\left(F_{0} \Lambda x, \Lambda x\right)=\psi_{0}\left(x^{*} x\right)=\sup _{i \in I} f_{i}\left(x^{*} x\right) \quad \text { for all } x \in \Re \text {. }
$$

In applying this result to the weight $\psi_{0}$ we get

$$
\psi_{0}(z)=\sup _{i \in I} f_{i}(z) \quad \text { for all } z \in \mathfrak{M}_{\psi_{0}}^{+} \quad \text { i.e. }
$$

for $z \in A^{+}$such that $\psi_{0}(z)<\infty$.

Applying Corollary 4.4 with trivial $G$ to any lower semi-continuous weight on $A$ such that $\overline{\mathfrak{M}}=A$ we get the existence of an increasing net $\left\{f_{i}\right\}_{i \in I} \subseteq \mathscr{F}$ such that $\varphi(z)=\sup _{i \in I} f_{i}(z)$ for all $z \in \mathfrak{M}^{+}$.

CoRollary 4.5. Let $\varphi$ be G-invariant weight on $A$ such that $\overline{\mathfrak{M}}=A$. Assume there is a family $\left\{f_{i}\right\}_{i \in I}$ in $\mathscr{F}$ such that

$$
\varphi(x)=\sum_{i \in I} f_{i}(x) \quad \text { for } x \in \mathfrak{M}^{+} .
$$

Then there is a family $\left\{f_{i}^{0}\right\}_{i \in I}$ in $\mathscr{F}_{0}$ such that $\psi_{0}(x)=\sum_{i \in I} f_{i}^{0}(x)$ for all $x \in \mathfrak{M}^{+}$.

Proof. By Lemma 2.6 we get operators $T_{i} \in \mathscr{C}^{*} \mathscr{K}$ such that

$$
\varphi\left(x^{*} x\right)=\sum_{i \in I}\left(T_{i} \Lambda x, \Lambda x\right)=(\Lambda x, \Lambda x) \quad \text { for } x \in \Re \text {. }
$$

So that $I=\sum_{i \in I} T_{i}$. As $\varphi$ is lower semi-continuous we have $F=I$ by Corollary 4.2 and $\dot{\phi}(I)=F_{0}$ by Corollary 3.3 .

So by the normality of $\dot{\phi}$ we get

$$
F_{0}=\sum_{i \in I} \phi\left(T_{i}\right)
$$

and so

$$
\begin{aligned}
\psi_{0}\left(x^{*} x\right)=\left(F_{0} \Lambda x, \Lambda x\right) & =\sum_{i \in I}\left(\phi\left(T_{i}\right) \Lambda x, \Lambda x\right) \\
& =\sum_{i \in I} \phi^{\prime}\left(f_{i}\right)\left(x^{*} x\right)
\end{aligned}
$$

for $x \in \mathfrak{R}$

by the use of Proposition 3.4. As $\phi^{\prime}\left(f_{i}\right) \in \mathscr{F}_{0}$. we proved the corollary. 
REMARK 4.6. In Theorem 4.1, as well as in the Corollaries 4.4 and 4.5 we find that two lower semi-continuous weights coincide on $\mathfrak{M}^{+}$. It is not yet known if this implies that they will coincide on all of $A([1]$, p. 74). However if we assume the existence of a two sided ideal $\mathfrak{R}_{1}$ contained in $\mathfrak{N}$ and dense in $A$ then this weights will coincide everywhere. Indeed there exists an approximate identity $\left\{u_{\lambda}\right\}$ in $\mathfrak{N}_{1}^{*} \mathfrak{R}_{1}$ for $A$. So for any $z \in A^{+},\left\{z^{1 / 2} u_{2} z^{1 / 2}\right\}$ is a net in $\mathfrak{M}^{+}$ tending to $z$ from below. (see also [8], Cor. 3.2)

Proposition 4.7. Let $\varphi$ be a G-invariant weight on $A$ such that $\mathfrak{M}$ is norm dense in $A$. For any $G$-invariant $T \in \pi(A)^{\prime}$ such that $0 \leqslant T \leqslant F_{0}$ there is a G-invariant weight $\psi$ such that $\psi \leqslant \psi_{0}$ and $\psi\left(x^{*} x\right)=(T \Lambda x, \Lambda x)$ for all $x \in \mathfrak{N}$. For any G-invariant weihgt $\psi$ such that $\psi \leqslant \psi_{0}$ and $\psi$ is lower semi-continuous on $\mathfrak{M}^{+}, \psi \mid \mathfrak{M}^{+}$is the upper envelope of a family of G-invariant continuous positive linear functionals. For any weight $\psi$ on $A$ such that $\psi \leqslant \phi$ and i $\mid \mathfrak{M}^{+}$is the upper envelope of G-invariant continuous positive linear functionals there exists an operator $T \in \pi(A)^{\prime}$ such that $T$ is invariant, $0 \leqslant T \leqslant F_{0}$ and $\psi\left(x^{*} x\right)=(T \Lambda x, \Lambda x)$ for $x \in \mathfrak{R}$.

Proof. First let $T \in \pi(A)^{\prime}$ such that $0 \leqslant T \leqslant F_{0}$. Define the function $\psi$ on $A^{+}$by

$$
\begin{array}{rlr}
\psi(x) & =\left(T \Lambda x^{1 / 2}, \Lambda x^{1 / 2}\right) & \text { for } x \in \mathfrak{M}^{+} \\
& =\infty & \text { for } x \in A^{+}, x \notin \mathfrak{M}^{+}
\end{array}
$$

Clearly $\psi(\lambda x)=\lambda \psi(x)$ for all real $\lambda>0$ and

$$
\psi(x) \leqslant\left\|F_{0} \Lambda x^{1 / 2}\right\| \leqslant\left\|\Lambda x^{1 / 2}\right\|=\varphi(x) \quad \text { for } x \in \mathfrak{M}^{+} .
$$

We prove that $\psi(x+y)=\psi(x)+\psi(y)$ for all $x, y \in A^{+}$. It is clearly sufficient to show it for $x, y \in \mathfrak{M}^{+}$. As $F_{0} \leqslant F$ we have $T \leqslant F$ and

$$
T \in F \pi^{\prime}(A) F=\overline{\mathscr{K}}^{*} \overline{\mathscr{K}}=\overline{\mathscr{K}^{*}} * \overline{\mathscr{K}} \quad \text { by ([3] Lemma 2.2). }
$$

So $T$ is weakly adherent to elements of the form

$$
\sum_{i=1}^{n} S_{i}^{*} T_{i} \quad \text { with } S_{i}, T_{i} \in \mathscr{\mathscr { C }} \text {. }
$$

But $\psi(x)=\left(T \Lambda x^{1 / 2}, \Lambda x^{1 / 2}\right)$ and if $\alpha_{i}, \beta_{i}$ are the vectors in $\mathscr{C}$ corresponding to $S_{i}$ and $T_{i}$ (Lemma 2.3) we also have that

$$
\left(\sum_{i=1}^{n} S_{i}^{*} T_{i} \Lambda x^{1 / 2}, \Lambda x^{1 / 2}\right)=\sum_{i=1}^{n}\left(\pi(x) \beta_{i}, \alpha_{i}\right) .
$$

So given $x, y \in \mathfrak{M}^{+}$we can find vectors $\alpha_{i}, \beta_{i} \in \mathscr{H}$ such that 


$$
\left|\psi(z)-\sum_{i=1}^{n}\left(\pi(z) \beta_{i}, \alpha_{i}\right)\right|<1
$$

for $z$ equal to $x, y$ or $x+y$. So we get $|\psi(x+y)-\psi(x)-\psi(y)|<3$ and by homogenuity that $\psi(x+y)=\psi(x)+\psi(y)$. If moreover $T$ is $G$-invariant then $\psi$ is clearly $G$-invariant. So we proved the first part of the proposition.

Let $\psi$ be a $G$-invariant weight, majorized by $\varphi$ and lower semicontinuous on $\mathfrak{M}^{+}$, then by ([1] Prop. 1.7) $\psi \mid \mathfrak{M}^{+}$is weakly adherent to the family of positive continuous linear functionals majorized by $\psi$. So by ([1] Lemma 2.6) $T$ is weakly adherent to the family

$$
\left\{S \in \mathscr{\Upsilon}^{*} \mathscr{\mathscr { C }}\{0 \leqslant S \leqslant T\}\right.
$$

where $T$ is the operator in $\pi(A)^{\prime}$ such that $\psi\left(x^{*} x\right)=(T \Lambda x, \Lambda x)$ for $x \in \mathfrak{R}$ ([1] Lemma 2.3). So we may apply $\phi$ and use its normality to get that $\phi(T)$ is weakly adherent to

$$
\left\{\dot{\phi}(S)\left\{S \in \mathscr{K}^{*} \mathscr{K}, 0 \leqslant S \leqslant T\right\}\right. \text {. }
$$

But as $\psi$ is $G$-invariant, so is $T$ and $\phi(T)=T$. Also $\phi\left(\mathscr{K}^{*} \mathscr{K}\right)=$ $\mathscr{K}_{0}^{*} \mathscr{K}_{0}$ so that $T$ is weakly adherent to

$$
\left\{S_{0}\left\{S_{0} \in \mathscr{K}_{0}^{*} \mathscr{K}_{0}, 0 \leqslant S_{0} \leqslant T\right\}\right. \text {. }
$$

Again by ([1] Lemma 2.6) we have that $\psi_{0} \mid \mathfrak{M}^{+}$is weakly adherent to the family of $G$-invariant continuous positive linear functionals majorized by $\psi_{0}$.

To prove the third part, let $\psi$ be a weight such that $\psi \leqslant \varphi$ and $\psi \mid \mathfrak{M}^{+}$is the upper envelope of $G$-invariant continuous positive linear functionals. By ([1] Lemma 2.3) there is a $T \in \pi(A)^{\prime}$ such that $0 \leqslant T \leqslant 1$ and $\psi\left(x^{*} x\right)=(T \Lambda x, \Lambda x)$ for $x \in \mathfrak{R}$. Then again by ([1] Lemma 2.6) $T$ is weakly adherent to the family

$$
\left\{S \in \mathscr{\varkappa}_{0}^{*} \mathscr{K}_{0}\{0 \leqslant S \leqslant T\} .\right.
$$

So $T \in \overline{\mathscr{K}}_{0} * \overline{\mathscr{K}}_{0}=\overline{\mathscr{K}}_{0}^{*} \overline{\mathscr{K}}_{0}$ by ([3] Lemma 2.2$)$. It follows that

$$
T=F_{0} T F_{0} \leqslant F_{0} \text {. }
$$

Corollary 4.8. Let $\varphi$ be any weight on $A$ such that $\overline{\mathfrak{M}}=A$. Given $T \in \pi(A)^{\prime}$ such that $0 \leqslant T \leqslant F$ there exists a weight $\psi \leqslant \varphi$ such that $\psi\left(x^{*} x\right)=(T \Lambda x, \Lambda x)$ for all $x \in \mathfrak{R}$.

Proof. Apply the fiirst part of Proposition 4.7 to the case where $G$ is trivial. 
REMARKS 4.9. Corollary 4.8 is in a sense the inverse of ([1] Lemma 2.3). On the other hand Lemma 2.6 shows a similar relation for the set $\left\{S \xi S \in \mathscr{K}^{*} \mathscr{K}, 0 \leqslant S \leqslant 1\right\}$ and the set of continuous positive linear functionals majorized by $\varphi$. One may ask if for all $T \in \pi(A)^{\prime}$ such that $0 \leqslant T \leqslant 1$ there exists a weight $\psi$ such that $\psi \leqslant \varphi$ and $\psi\left(x^{*} x\right)=(T \Lambda x, \Lambda x)$ for all $x \in \mathfrak{N}$. It can be shown to be true if $A$ is a von Neumann algebra. Indeed the only trouble is to show that the function $\psi$ defined on $A^{+}$by $\psi(x)=\left(T \Lambda x^{1 / 2}, \Lambda x^{1 / 2}\right)$ if $x \in \mathfrak{M}^{+}$and $\psi(x)=\infty$ if $x \in A^{+}$but $x \notin \mathfrak{M}^{+}$satisfies

$$
\psi(x+y)=\psi(x)+\psi(y) \text {. }
$$

If now $A$ is a von Neumann algebra we can again find operators $u$ and $v \in A$ such that

(i ) $x^{1 / 2}=u(x+y)^{1 / 2}, y^{1 / 2}=v(x+y)^{1 / 2}$

(ii) $\left(u^{*} u+v^{*} v\right)(x+y)^{1 / 2}=(x+y)^{1 / 2}$, see proof of ([5] Th. 1 p. 85). So that

$$
\begin{aligned}
\psi(x)+\psi(y)= & \left(T \Lambda x^{1 / 2}, \Lambda x^{1 / 2}\right)+\left(T \Lambda y^{1 / 2}, \Lambda y^{1 / 2}\right) \\
= & \left(T \Lambda(x+y)^{1 / 2}, \pi\left(u^{*} u\right) \Lambda(x+y)^{1 / 2}\right) \\
& +\left(T \Lambda(x+y)^{1 / 2}, \pi\left(v^{*} v\right) \Lambda(x+y)^{1 / 2}\right) \\
= & \left(T \Lambda(x+y)^{1 / 2}, \Lambda(x+y)^{1 / 2}\right)=\psi(x+y)
\end{aligned}
$$

for $x, y \in \mathfrak{M}^{+}$.

\section{EXAMPLes.}

5.1. Let $G$ be a compact group acting as *automorphisms on a von Neumann algebra $A$. Assume that the function $g \rightarrow g(x)$ is strongly continuous for all $x \in A$. Let $\varphi$ be a $G$-invariant ultra-weakly lower semi-continuous weight on $A$ such that $\mathfrak{M}$ is ultra-weakly dense in $A$. We will show that $\varphi \mid \mathfrak{M}$ is weakly adherent to the set of $G$ invariant normal functionals majorized by $\varphi$. Consider $T \in \mathscr{K}^{*} \mathscr{K}^{\mathscr{T}}$, there exist $S_{i}, T_{i} \in \mathscr{C}$ such that $T=\sum_{i=1}^{n} S_{i}^{*} T_{i}$. By Lemma 2.3 there exists vectors $\alpha_{i}, \beta_{i} \in \mathscr{H}$ such that $S_{i} \Lambda x=\pi(x) \alpha_{i}$ and $T_{i} \Lambda x=$ $\pi(x) \beta_{i}$ for all $x \in \mathfrak{N}$. For all $g \in G$ and $x, y \in \mathfrak{R}$ we have

$$
\left(U_{g}^{-1} T U_{g} \Lambda x, A y\right)=\sum_{i=1}^{n}\left(\pi\left(g\left(y^{*} x\right)\right) \beta_{i}, \alpha_{i}\right) \text {. }
$$

By the normality of $\pi$ ([10] Prop. 13.10) we have that the function $g \rightarrow U_{g}^{-1} T U_{g}$ is weakly continuous.

So we can define for all $T \in \mathscr{K}^{*} \mathscr{K}$ an operator $\phi_{1}(T)$ by

$$
\phi_{1}(T)=\int_{G} U_{g}^{-1} T U_{g} d g
$$


where $d g$ is the normalized Haar measure on $G$.

It is clear that $\phi_{1}$ is a linear positive $G$-invariant map into the fixed points of $\pi(A)^{\prime}$. Consider now also the projection map $\phi$ of Proposition 3.2. As $\varphi$ is ultra-weakly lower semi-continuous, $\mathscr{K}^{*} \mathscr{K}$ is dense in $\pi(A)^{\prime}$ (Corollary 4.2) and $\phi$ is defined on $\pi(A)^{\prime}$. By normality and $G$-invariance of $\phi$ we get

$$
\dot{\phi}\left(\dot{\phi}_{1}(T)\right)=\int_{G} \phi\left(U_{g}^{-1} T U_{g}\right) d g=\dot{\phi}(T) .
$$

On the other hand $\phi\left(\phi_{1}(T)\right)=\phi_{1}(T)$ because $\phi$ is a projection map onto the fixed points of $\pi(A)^{\prime}$. Therefore $\phi_{1}=\phi \mid \mathscr{K}^{*} \mathscr{K}$. Let $S_{\alpha}$ be an increasing net of positive elements in $\mathscr{K}^{*} \mathscr{K}$ tending to $I$. Clearly by the definition of $\dot{\phi}_{1}$ we will have that $\dot{\phi}_{1}\left(S_{\alpha}\right) \rightarrow I$. On the other hand $\phi_{1}\left(S_{\alpha}\right)=\phi\left(S_{\alpha}\right)$ and $\phi$ is normal so that $\phi\left(S_{\alpha}\right) \rightarrow \phi(I)$. By Corollary 4.2 we have $F=I$ and by Corollary 3.2 that $\phi(F)=F_{0}$. It follows at once that $F_{0}=I$ and by Theorem 4.1 we get that $\varphi\left|\mathfrak{M}=\psi_{0}\right| \mathfrak{M}$ where $\psi_{0}$ is the upper envelope of normal $G$-invariant continuous linear functionals.

5.2. In our first example we found that the weight $\varphi$ was the upper envelope of invariant normal functionals. It is not hard to find an example for the other extreme. Let $A$ be a semi-finite von Neumann algebra with no finite portion, i.e., with no finite nonzero central projection. Let $\phi$ be a faithful normal semi-finite trace on $A$. If $G$ is the group of all inner automorphisms, then $\phi$ is a $G$ invariant $\sigma$-weakly lower semi-continuous weight on $A$ and $\mathfrak{M}$ is $\sigma$ weakly dense.

Since $A$ is properly infinite there are no finite normal traces on $A$. The weight $\psi_{0}$ constructed in Theorem 2.10 is the upper envelope of normal finite traces majorized by $\varphi$, hence $\psi_{0}=0$.

\section{REFERENCES}

1. F. Combes, Poids sur une $C^{*}$-algèbre, J. Math. Pures et Appl., 47, (1968), 57-100.

2. - Poids associé à une algèbre hilbertienne à gauche, Compositio Mathematica, 23, (1971), 49-77.

3. - Poids et espérances conditionelles dans les algèbres de von Neumann, Bull. Soc. Math. France, 99 (1971), 73-112.

4. F. Combes and F. Perdrizet, Ideaux dans les expaces vectoriels ordonnés, J. Math. Pures et Appl., 49 (1970), 29-59,

5. J. Dixmier, Les algèbres d'opérateurs dans l'expace Hilbertien, $2^{\circ}$ édition, Paris Villars 1969 .

6. S. Doplicher, D. Kastler, and E. St $\phi$ rmer, Invariant states and asymptotic abelianness, J. Funct. Anal., 3 (1969), 419-434.

7. I. Kaplansky, $A$ theorem on rings of operators, Pacific J. Math., 1, (1951), 227-232.

8. G. K. Pedersen, Measure theory for $C^{*}$-algebras, Math. Scand., 19 (1966), 131-145. 
9. F. Riesz and Sz. Nagy, Leçons d'analyse fonctionelle, Akadémiai Kiado Budapest, 1952.

10. M. Takesaki, Lecture notes U.C.L.A. on operator algebras.

Received January 6, 1972. On leave from the University of Leuven (Belgium); aangesteld navorser bÿ het Belgisch N.F.W.O.

Present address: School of Mathematics, University of Newcastle Upon Tyne, Newcastle Upon Tyne NEl 7RU, England. 


\section{PACIFIC JOURNAL OF MATHEMATICS}

\section{EDITORS}

D. Gilbarg and J. Milgram Stanford University

Stanford, California 94305

R. A. Beaumont

University of Washington

Seattle, Washington 98105
J. DugundJI

Department of Mathematics

University of Southern California

Los Angeles, California 90007

RICHARD ARENS

University of California

Los Angeles, California 90024

ASSOCIATE EDITORS
E. F. BECKENBACH
B. H. NeumanN
F. WOLF
K. YoSHIDA

\section{SUPPORTING INSTITUTIONS}

UNIVERSITY OF BRITISH COLUMBIA

CALIFORNIA INSTITUTE OF TECHNOLOGY

UNIVERSITY OF CALIFORNIA

MONTANA STATE UNIVERSITY

UNIVERSITY OF NEVADA

NEW MEXICO STATE UNIVERSITY

OREGON STATE UNIVERSITY

UNIVERSITY OF OREGON

OSAKA UNIVERSITY
UNIVERSITY OF SOUTHERN CALIFORNIA

STANFORD UNIVERSITY

UNIVERSITY OF TOKYO

UNIVERSITY OF UTAH

WASHINGTON STATE UNIVERSITY

UNIVERSITY OF WASHINGTON

$\stackrel{*}{*} \stackrel{*}{*} \stackrel{*}{*}$ AMERICAN MATHEMATICAL SOCIETY

NAVAL WEAPONS CENTER 


\section{Pacific Journal of Mathematics}

\section{Vol. 46, No. $1 \quad$ November, 1973}

Allan Francis Abrahamse, Uniform integrability of derivatives on $\sigma$-lattices .......................................... 1

Ronald Alter and K. K. Kubota, The diophantine equation $x^{2}+D=p^{n} \ldots \ldots \quad 11$

Grahame Bennett, Some inclusion theorems for sequence spaces .......... 17

William Cutler, On extending isotopies ........................ 31

Robert Jay Daverman, Factored codimension one cells in Euclidean

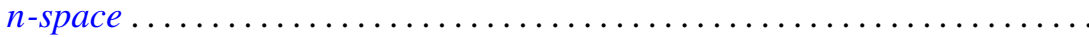

Patrick Barry Eberlein and Barrett O’Neill, Visibility manifolds ............ 45

M. Edelstein, Concerning dentability .......................... 111

Edward Graham Evans, Jr., Krull-Schmidt and cancellation over local

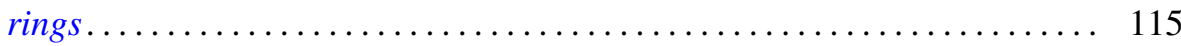

C. D. Feustel, A generalization of Kneser's conjecture ................ 123

Avner Friedman, Uniqueness for the Cauchy problem for degenerate parabolic equations .......................................... 131

David Golber, The cohomological description of a torus action ............ 149

Alain Goullet de Rugy, Un théorème du genre "Andô-Edwards" pour les

Fréchet ordonnés normaux..............................

Louise Hay, The class of recursively enumerable subsets of a recursively enumerable set ........................................

John Paul Helm, Albert Ronald da Silva Meyer and Paul Ruel Young, On orders of translations and enumerations...

Julien O. Hennefeld, A decomposition for $B(X)^{*}$ and unique Hahn-Banach

extensions

Gordon G. Johnson, Moment sequences in Hilbert space .

Thomas Rollin Kramer, A note on countably subparacompact spaces ...

Yves A. Lequain, Differential simplicity and extensions of a derivation ....

Peter Lorimer, A property of the groups Aut $\mathrm{PU}\left(3, q^{2}\right) \ldots$

225

Yasou Matsugu, The Levi problem for a product manifold.

231

John M.F. O'Connell, Real parts of uniform algebras ......

William Lindall Paschke, A factorable Banach algebra without bounded

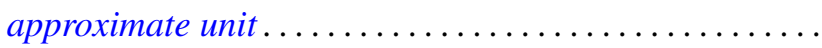

Ronald Joel Rudman, On the fundamental unit of a purely cubic field ....

Tsuan Wu Ting, Torsional rigidities in the elastic-plastic torsion of simply connected cylindrical bars .........................

Philip C. Tonne, Matrix representations for linear transformations on analytic sequences...................................

Jung-Hsien Tsai, On E-compact spaces and generalizations of perfect

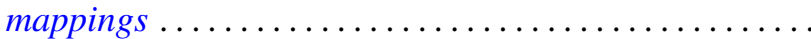

Alfons Van Daele, The upper envelope of invariant functionals majorized by an invariant weight. .. 